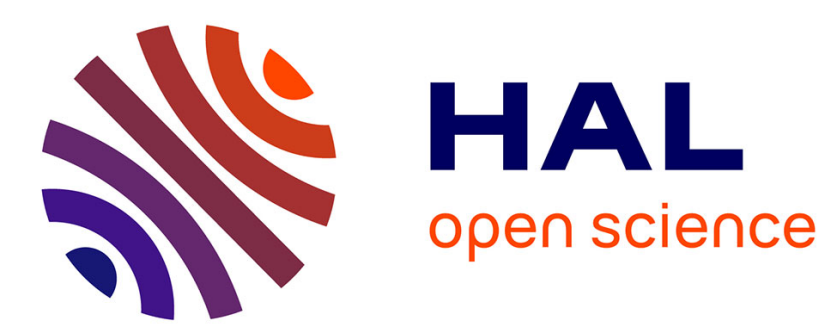

\title{
Duct modal detection tool to characterize the noise source generated by an air pump
}

\author{
Sandrine Fauqueux, Renaud Davy, Fabien Mery
}

\section{To cite this version:}

Sandrine Fauqueux, Renaud Davy, Fabien Mery. Duct modal detection tool to characterize the noise source generated by an air pump. 25th AIAA/CEAS Aeroacoustics Conference, May 2019, DELFT, Netherlands. 10.2514/6.2019-2419 . hal-02333572

\section{HAL Id: hal-02333572 \\ https://hal.science/hal-02333572}

Submitted on 25 Oct 2019

HAL is a multi-disciplinary open access archive for the deposit and dissemination of scientific research documents, whether they are published or not. The documents may come from teaching and research institutions in France or abroad, or from public or private research centers.
L'archive ouverte pluridisciplinaire HAL, est destinée au dépôt et à la diffusion de documents scientifiques de niveau recherche, publiés ou non, émanant des établissements d'enseignement et de recherche français ou étrangers, des laboratoires publics ou privés. 


\title{
Duct modal detection tool to characterize the noise source generated by an air pump
}

\author{
S. Fauqueux*, R. Davy ${ }^{\dagger}$ \\ French Aerospace Lab (ONERA), Aerodynamics Aeroelasticity Acoustics Department, Avenue de la Division Leclerc, \\ 92322 Châtillon Cedex, France \\ and F. Méry \\ French Aerospace Lab (ONERA), Multi-Physics Department for Energy, Université de Toulouse, F-31055, Toulouse, \\ France
}

\begin{abstract}
Aircraft air conditioning system must be compliant with the Noise Regulations of every countries in which it will be operated. In the Clean Sky 2 project IDEAS, the system of interest is composed of an air pump, developped by Liebherr Aerospace, that generates low and mid-frequency noise that may be mitigated by the use of innovative acoustic liners. The aim of this study is to assess the level and modal content of the generated noise source in two industrial operating conditions. The modal detection solution proposed by ONERA leads to the post-treatments of measurements obtained by a flush-mounted microphone array located downstream of the diffuser. As microphones will be implemented in a hard-wall region of the duct, the modal basis characterizing the measured acoustic field does not depend on the lined walls, located in the diffuser area.

The deconvolution method ARMADA leads to the minimization of the discrepancy, in the least-square sense, between measurements and an analytical model of the acoustic pressure field that propagates in cylindrical ducts. In the first part of this article, the analytical models in uniform and non-uniform flows are introduced, with underlying hypotheses. In the case of shear flow, the modal shapes are given by ONERA's MAMOUT code. Then, the deconvolution method is presented, considering that the modes are correlated, as wave reflections may occur at the end of Liebherr's bench. In the second part, the design of the IDEAS microphone array is defined, considering uniform flow only. Different sensor arrangements are compared. One design is retained and the associated estimation errors are assessed. In the third part, the impact of estimating modes under the uniform flow hypothesis despite the fact that shear flow occurs during measurements is evaluated. A potential misleading impact is highlighted at 3150 Hz on the $m=0$ and $n=1$ mode. Finally, the experimental setup is introduced and the analysis of the measurements performed in the facility without use of acoustic liners are detailed for two operating conditions.
\end{abstract}

\section{Introduction}

On modern aircraft, passengers and crew breathe a mixture of fresh and recirculated air. This combination rather than fresh only allows the regulation of temperature, pressure and humidity. The air is bled from the engines and supplied to air conditioning units. It is then ducted into the cabin, circulated and eventually drawn into the lower fuselage where it is sucked out by the pressurization outflow valve for the cycle to begin again. Besides creating a safe and comfortable environment, the aircraft air conditioning systems generate noise. This noise must be mitigated to comply with the local regulations on perceived noise for both the passengers and the airport workers when the aircraft is at the gate or is taxiing to the runway. As a consequence, any new generation of air conditioning system must be shown by the manufacturer to be compliant with the Noise Regulations of every countries in which it will be operated. The system of interest in the Clean Sky 2 project IDEAS, and developped by Liebherr Aerospace, is composed of an air pump that generates low and mid-frequency noise. This noise is conducted through several ducts to the exterior of the

\footnotetext{
* Sandrine.Fauqueux@onera.fr.

†Renaud.Davy@onera.fr.

†abien.Mery@onera.fr.
} 
aircraft, thus contributing to ramp noise. Consequently, this new technology requires the use of innovative acoustic liners to mitigate the noise source. An accurate knowledge of the noise source is therefore needed.

ONERA recently developed a modal deconvolution method, called ARMADA [1], based on the iterative minimization of the discrepancy, in the least-square sense, between flush-mounted microphone measurements and an analytical model that represents the propagation between the sources and the measurements positions. A rigid and infinite cylindrical or annular duct of constant radius subjects to a uniform and subsonic flow along the revolution axis of the duct is currently considered. In the industrial operating conditions of the air-pump of interest, most of these hypotheses are satisfied, except that the flow may potentially be sheared. A modal detection network that ensures the efficiency of the modal detection process, and suited to the geometry and constraints of the installation, has also to be defined.

In the first part of this article, the modal deconvolution method under uniform flow hypotheses is briefly presented before being extended to sheared flow. The second part is dedicated to the definition of the position of the microphones that ensures the good efficiency of the deconvolution method at the operating points. In the third part of this article, the influence of shear flow in our modal detection tool at one specific operating point is studied. Finally, the measurements performed in the facility without use of acoustic liners are introduced. Measurements with acoustic liners will be performed in the near future.

\section{Description of the deconvolution method under uniform and sheared flow hypotheses}

The modal detection network will be implemented in a hard-wall region of the duct with circular and constant section, located downstream of the diffuser. Reflections may occur at the end of the duct, as termination is not equipped with acoustic treatment. The flow is perturbed by sound coming from inside the pipe but perturbations amplitudes are considered to be small enough for linearisation. The acoustic noise of interest is a broad band one, mostly energetic between 300 and $2000 \mathrm{~Hz}$.

In the IDEAS project framework, two different flows are considered. An axisymmetric compressible RANS computation has been driven by Liebherr Aerospace under these two conditions. It is assumed that the mean flow is perfectly axisymmetric with only an axial component. The flow is clearly sheared in the radial direction, even if the shear is much less pronounced than what can be observed in the mixing region. The RANS flow solution was post-treated to assess the equivalent mean temperature, flow velocity and density at each axial location, by averaging over the cross-section. It has been ensured that the same mass flow is kept whatever the axial position is. This leads to the two equivalent uniform flow conditions:

- PT1: Speed of sound $352 \mathrm{~m} \cdot \mathrm{s}^{-1}$ and Mach 0.15

- PT2: Speed of sound $380 \mathrm{~m} \cdot \mathrm{s}^{-1}$ and Mach 0.16

We thereby consider a rigid and infinite cylindrical duct, of radius $R=86.5 \mathrm{~mm}$, subjects to a uniform or shear subsonic flow along the revolution axis of the duct $(0 x)$. The Fourier transform of the acoustic pressure field at frequency $v$, defined by:

$$
\hat{p}(\mathbf{x}, v)=\int_{-\infty}^{+\infty} p(\mathbf{x}, t) e^{-2 \mathrm{i} \pi v t} d t
$$

and propagating inside such a duct can be expressed as an infinite sum of azimuthal and radial modes:

$$
\hat{p}(x, r, \theta, v)=\sum_{m=-\infty}^{+\infty} \sum_{n=0}^{n=+\infty} \sum_{\varepsilon= \pm} A_{m, n}^{\varepsilon}(v) f_{m, n}(r) e^{-\mathrm{i} m \theta} e^{-\mathrm{i} k_{m, n}^{\varepsilon}(v) x}
$$

$m \in \mathbb{Z}$ and $n \in \mathbb{N}$ are called the azimuthal modes and radial modes respectively and $A_{m, n}^{\varepsilon}(v)$ is the mode amplitude. $\varepsilon=+$ and $\varepsilon=-$ are related to waves propagating downstream and upstream respectively. $(r, \theta)$ is the polar coordinate system of the duct section (see Fig. 11). In practice, the sum is finite. $N_{m o d e}^{\omega}$ denotes the number of modes that are considered in 22), defining the modal projecting base. 


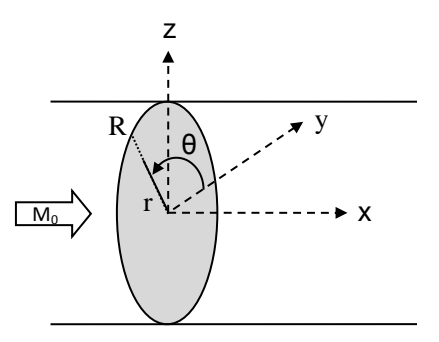

Fig. 1 In duct orthonormal coordinate system.

\section{A. Modal base in the case of uniform flow}

In case of uniform flow of Mach number $M_{0}, f_{m, n}$ and $k_{m, n}^{\varepsilon}$ can be analytically expressed [2]:

$$
\begin{array}{r}
f_{m, n}(r)=J_{m}\left(\mu_{m, n} \frac{r}{R}\right) \\
k_{m, n}^{\varepsilon}(v)=\frac{1}{\beta_{0}^{2}}\left[-M_{0} k_{0}(v)+\varepsilon \sqrt{k_{0}^{2}(v)-\beta_{0}^{2} \frac{\mu_{m, n}^{2}}{R^{2}}}\right]
\end{array}
$$

where $J_{m}$ denotes the Bessel functions of the first kind and $\mu_{m, n}$ is the $(n+1)^{\text {th }}$ root of $J_{m}^{\prime}$. $\beta_{0}$ and $k_{0}(v)$ are defined as:

$$
\beta_{0}^{2}=1-M_{0}^{2} \quad \text { and } \quad k_{0}(v)=\frac{2 \pi v}{c_{0}}
$$

and $c_{0}$ denotes the speed of sound. For mode of azimuthal order $m$ and radial order $n$ such that:

$$
\beta_{0} \mu_{m, n} \leq R k_{0}(v)
$$

$k_{m, n}^{\varepsilon}(v)$ takes real value and the mode is cut-on. Otherwise, the imaginary part of $k_{m, n}^{\varepsilon}(v)$ takes non zero value and the mode is cut-off.

\section{B. Modal base in the case of shear flow}

In order to take into account the shear flow effect on the acoustic propagation, let us consider an inviscid perfect gas flowing along an infinitely long cylindrical duct of radius $R$. Let $U_{x}, U_{r}$ and $U_{\theta}$ be the axial, radial and azimuthal components of the velocity vector $\mathbf{U}, \rho$ the density and $s$ the entropy per unit mass. $\gamma=C_{p} / C_{v}$ represents the ratio of specific heat capacities at constant pressure and constant volume respectively. The pressure, the density and the absolute temperature $T$ satisfy the equation of state $p=\rho\left(C_{p}-C_{v}\right) T$. Then, if $D / D t$ stands for the convective derivation $\partial / \partial t+(\mathbf{U} \cdot \nabla)$, the Euler equations read:

$$
\begin{aligned}
\frac{\partial \rho}{\partial t}+\nabla \cdot(\rho \mathbf{U}) & =0 \\
\rho \frac{D \mathbf{U}}{D t}+\nabla p & =0 \\
\frac{D s}{D t} & =0
\end{aligned}
$$

The flow field is written as the sum of a steady base flow and a small-amplitude unsteady perturbation:

$$
(U, p, \rho)=\left(U, p_{0} / \gamma, \rho_{0}\right)+\left(u^{\prime}, p^{\prime}, \rho^{\prime}\right)
$$

The base flow is supposed to be streamwise-invariant and axisymmetric, which means that:

$$
U_{x}=U_{x}(r), \quad U_{r}=0, \quad U_{\theta}=0, \quad \rho_{0}=\rho_{0}(r)
$$


RANS results show that these assumptions are quite well verified in the cylindrical duct downstream of the diffuser. In order to obtain the linearized Euler equations, small amplitude disturbance fields is sought for under harmonic formation:

$$
\left(u_{x}^{\prime}, u_{r}^{\prime}, u_{\theta}^{\prime}, p^{\prime}, \rho^{\prime}\right)=\left(U_{x}^{\prime}, U_{r}^{\prime}, U_{\theta}^{\prime}, P^{\prime}, \Sigma^{\prime}\right) e^{\mathrm{i}(2 \pi v t-k x-m \theta)}
$$

where $\omega=2 \pi v$ is the pulsation, $k$ the complex wave number and $m$ the azimuthal wave number. The velocity, pressure and density disturbance $\left(U_{x}^{\prime}, U_{r}^{\prime}, U_{\theta}^{\prime}, P^{\prime}, \Sigma^{\prime}\right)$ are unknown functions of $r$. The entropy equation simplifies the system and assumes that $P=\Sigma$, the linearised Euler equations for mass and momentum can be written as follows:

$$
L(k, \omega) \cdot X=0
$$

where $L$ is a linear operator and $X=\left(P^{\prime}, U_{x}^{\prime}, U_{r}^{\prime}, U_{\theta}^{\prime}\right)$. $m$ is fixed and $\omega$ and $k$ must be fit to consider either a spatial or a temporal eigenvalue problem (usually, for acoustics issues, a spatial eigenvalue problem is considered, i.e. $v$ is a given real frequency). Concerning the boundary conditions, the duct wall can be constituted of either a locally reacting liner of complex specific impedance $Z$ or a rigid wall $\left(Z \rightarrow \infty\right.$ or $U_{r}^{\prime}=0$ at the wall). This boundary satisfies Ingard-Myers condition [3, 4]. Solving this linear problem with the boundary condition for a fixed azimuthal wavenumber $m$, a known mean velocity and density profile and impedance $Z$ implies solving a dispersion relation:

$$
D(k, \omega, m)=0
$$

This generalized eigenvalue problem can be solved thanks to the MAMOUT code [5], in which the equations are discretized along the radial direction through a spectral collocation method (Tchebychech polynomials) or through a compact finite-difference scheme (Gamet scheme, of order 6 on a regular mesh). $\omega$ is a real number fixed by the considered frequency. $k$ is obtained thanks to the dispersion relation solution, and is a complex number. For each eigenvalue $k$, the associated $X$ eigenvector is also computed. The resolution of such an eigenvalue problem enables to compute cut-on modes but also cut-off modes, in both directions of propagation (downstream and upstream).

In the present configurations, shear flow has only significant effect on the wave numbers for left-running cut-on modes at high frequencies (over $2500 \mathrm{~Hz}$ ). Consequently, the simplified uniform flow framework may be sufficient for correctly catching the acoustic propagation within the IDEAS duct, and therefore for designing the modal detection ring. However, this point will be checked in section IV

\section{ARMADA deconvolution method}

In the following, only cut-on modes are considered, composing the modal projecting base. $N_{\text {mode }}^{\omega}$ denotes the number of cut-on modes at the frequency $v$. The analytical model of the Fourier transform of the acoustic pressure field being defined, either under uniform or shear flow, we can now briefly introduce our deconvolution method, called ARMADA (Azimuthal and Radial Modal Analysis for in-Duct Acoustics). See [1] for more details. We consider an antenna composed by $N$ flush-mounted microphones of coordinates $\left(x_{i}, R, \theta_{i}\right)_{i=1 . . N}$ in the coordinate system represented in Fig. 1 The power cross-spectral density is defined as the Fourier transform of the temporal cross-correlation function. The signal being centred, one can introduce the power cross-spectral matrix (PCSM) of the measured acoustic pressure field, estimated through Welch's method [6]:

$$
\Gamma_{i, j}^{\text {mesure }}(v)=\overline{\hat{p}\left(x_{i}, R, \theta_{i}, v\right) \hat{p}^{*}\left(x_{j}, R, \theta_{j}, v\right)}
$$

where $z^{*}$ is the complex conjugate of $z$ and $\bar{z}$ denotes the expected value on the different temporal data segments. In the same way, one can introduce the PCSM of the modes amplitude:

$$
\mathbf{S}_{\left[m_{i}, n_{i}, \varepsilon_{i}\right]\left[m_{j}, n_{j}, \varepsilon_{j}\right]}(v)=\overline{A_{m_{i}, n_{i}}^{\varepsilon_{i}}(v) A_{m_{j}, n_{j}}^{\varepsilon_{j}{ }^{*}}(v)}
$$

By using the theoretical expression of the measured acoustic pressures given by equation [2], an analytical model of the measured power PCSM is derived:

$$
\boldsymbol{\Gamma}^{\text {model }}(v)=\mathbf{G}(v) \mathbf{S}(v) \mathbf{G}^{\mathrm{H}}(v)
$$

$\mathbf{G}$ is the modal propagation matrix, defined as:

$$
\mathbf{G}_{i,\left[m_{j}, n_{j}, \varepsilon_{j}\right]}(v)=f_{m_{j}, n_{j}}(R) e^{-\mathrm{i} m_{j} \theta_{i}} e^{-\mathrm{i} k_{m_{j}, n_{j}}^{\varepsilon_{j}}(v) x_{i}}
$$


and $\mathbf{G}^{\mathrm{H}}$ is the conjugate transposed matrix of $\mathbf{G}$. Our modal deconvolution consists in estimating $\mathbf{S}$ by solving the following minimization problem in the least square sense:

$$
\tilde{\mathbf{S}}(v)=\arg \min _{\mathbf{S}(v)}\left\|\boldsymbol{\Gamma}^{\text {mesure }}(v)-\mathbf{G}(v) \mathbf{S}(v) \mathbf{G}^{\mathrm{H}}(v)\right\|_{\mathrm{F}}^{2}
$$

with $\|\cdot\|_{\mathrm{F}}$ the Frobenius matrix norm. $\mathbf{S}(v)$ being a power cross spectral matrix, it has to be a Hermitian, positive-semi definite matrix. The quadratic minimization problem (19) is iteratively resolved using a gradient descent algorithm considering that modes are uncorrelated (i.e: $\mathbf{S}(v)$ is a diagonal matrix at each frequency $v$ ), or correlated (i.e : $\mathbf{S}(v)$ is a full matrix at each frequency $v$ ). See [7] for more details on the iterative process. Non-linear constraint is imposed by projection during the iterative process, and the minimization algorithm is performed frequency by frequency, independently. None regularization scheme is currently used. In Liebherr Aerospace's facility, the deconvolution method is applied under the correlated modes assumption, as reflections may occur at the end of the duct that imply correlation between downstram and upstream modes at least [1].

\section{Microphone array design}

The operating condition and deconvolution method being defined, we now have to design a microphone network adapted to the installation constraints. The acoustic noise of interest is a broad band one, mostly energetic between 300 and $2000 \mathrm{~Hz}$. The deconvolution method is applied at central frequency of third octave bands, from $200 \mathrm{~Hz}$ to $3150 \mathrm{~Hz}$. A number of approximatively 30 microphones are owned by Liebherr Aerospace to design the flush mounted microphone array.

In this frequency bandwidth and under the operating condition, the azimuthal order of cut-on modes varies from -3 to 3, with a maximum of 2 radial modes. First of all, two typical microphone array designs are considered (see 2). The first one is composed of one ring and a linear array. The second one is composed of two rings and a linear array, each part being discretized with a constant step. We use the numerical evaluation procedure presented in [1], §2, to determine the minimum amount of microphones and the space step of the linear array that enables a satisfying estimation of the modal content. For frequency greater or equal to $2000 \mathrm{~Hz}$, only the second design gives good estimation results. The linear array is composed of 7 microphones, with a space step of $0.025 \mathrm{~m}$, surrounded by 2 rings. Only 7 microphones would be needed on the ring if modes were uncorrelated but, as we assume that modes are correlated, we decided to over-sample the ring by using 12 or 13 microphones.
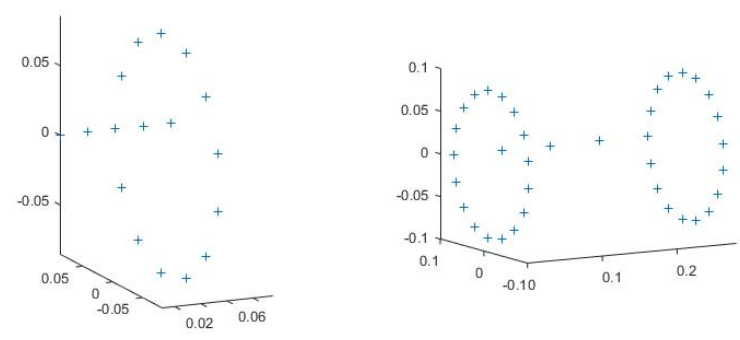

Fig. 2 Typical microphone array designs.

Based on these preliminary design, five additional microphone arrays are defined (see figure 3):

- Design 1: 2 rings of 13 microphones - 1 linear array of 7 regularly spaced microphones.

- Design 2: 1 ring of 13 microphones -1 helix of 13 microphones.

- Design 3: 2 rings of 13 microphones - 1 helix of 13 microphones.

- Design 4: 2 rings of 13 microphones - half an helix of 6 microphones.

- Design 5: 1 ring of 13 microphones -1 helix of 13 microphones -1 ring of 6 microphones.

- Design 6: 1 ring of 12 microphones -1 helix of 11 microphones - 1 ring of 6 microphones.

One way to choose the optimal design is to re-apply the numerical evaluation procedure to every design at each frequency. Another way is to evaluate the condition number of the matrix that has to be inversed when the deconvolution 

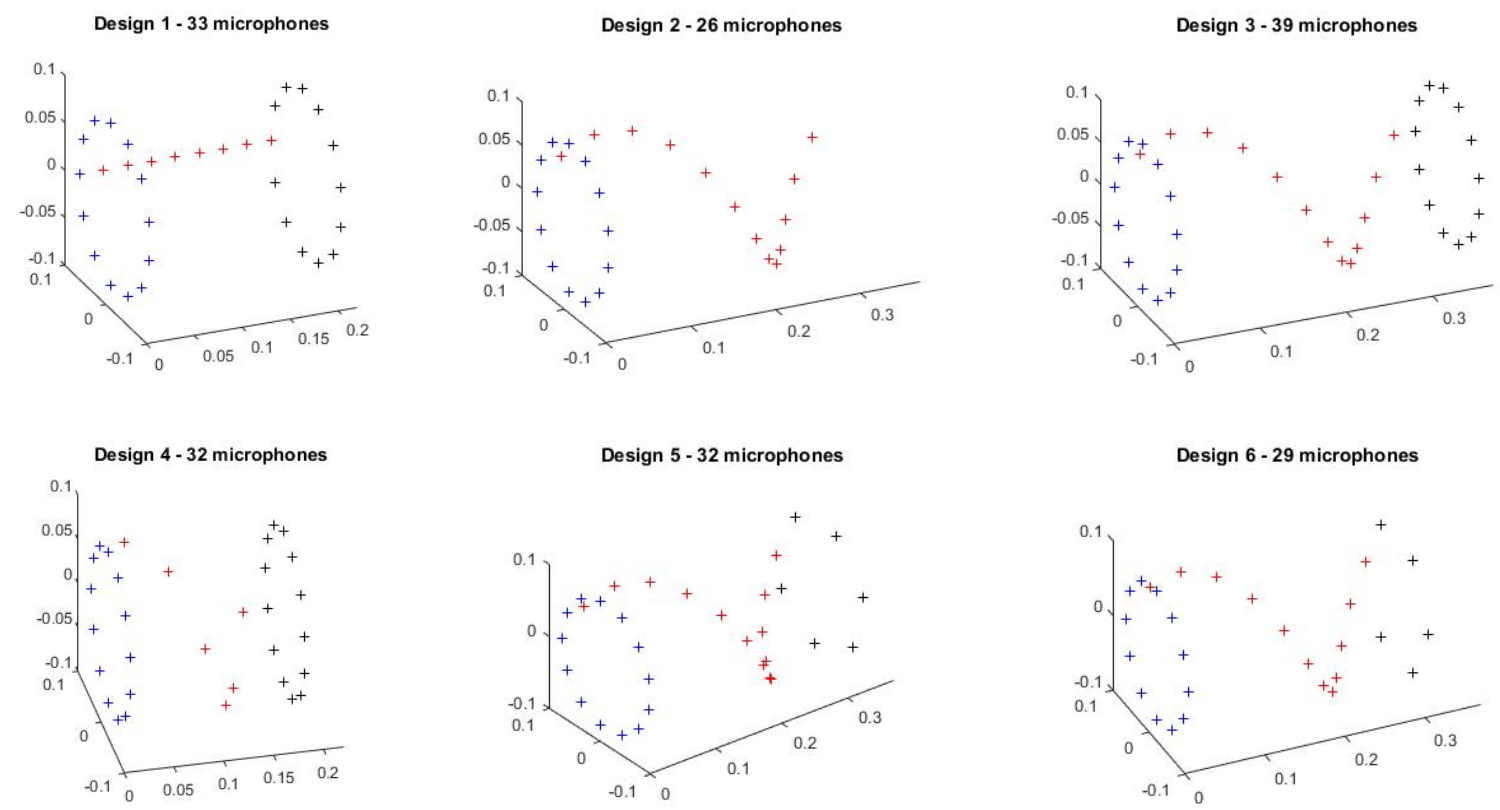

Fig. 3 Microphone array designs defined in this study.

problem is written as a linear one (see [1] for mode details). Indeed, the problem to solve, which is formulated by equation (19), can be seen as searching the solution of:

$$
\boldsymbol{\Phi}(v) \overline{\mathbf{S}}(v)=\overline{\boldsymbol{\Gamma}}^{\text {mesure }}(v)
$$

where, for every matrix $\mathbf{M}, \overline{\mathbf{M}}$ corresponds to a vectorial shape of $\mathbf{M}$, and $\boldsymbol{\Phi}(v)$ can be expressed as a function of $\mathbf{G}(v)$. $\boldsymbol{\Phi}(v)$ depends on the modal basis as well as on the microphones position. Thus, its condition number gives us information about the ill or well-posed property of the problem formulated by equation (19), even if neither the constraint, nor the algorithm take part in the study. Such condition analysis were exploited by U. Tapken and L. Enghardt in [8] to compare the suitability of different sensor arrangements, and choose their optimum number of axial and radial measurements positions. The condition number associated to each microphone array are represented in figure 4 . One first can notice that the condition number is improved by using an helix microphone array instead of a linear one. It is also better to design a microphone array with 2 rings (design 2 vs. design $3,4,5$ and 6 ). The $3^{\text {rd }}, 5^{\text {th }}$ and $6^{\text {th }}$ designs give the best condition numbers. As the amount of microphones of the $6^{\text {th }}$ design met the Liebherr Aerospace's request, this microphone array is selected.

Finally, the numerical evaluation procedure is applied at the central frequency of third octave band between 200 $\mathrm{Hz}$ and $3150 \mathrm{~Hz}$, considering the microphone array design 6 and the two operating points condition. A statistical information is obtained with 100 realizations of the simulated power-cross spectral matrix of the amplitude of modes (see [1], §2, for mode details). The estimation error is always smaller than $1 \%$ regardless the frequency.

\section{Influence of shear flow on the evaluation of the modal content of the acoustic pressure field by the deconvolution method}

Our deconvolution method needs the knowledge of the modal base characteristics (azimuthal, radial and longitudinal dependence) of the acoustic propagation encountered during the measurements. Axisymmetric compressible RANS simulations have shown that the flow is clearly sheared in the radial direction. As presented in section II, the modal base may be obtained under uniform as well as shear flow (under certain assumptions). However, getting the characteristics in the shear flow case requires the knowledge of the flow profile and a pre-computation by using MAMOUT code. Thus, it is of interest to determine the configurations (frequency among other) where the approximation by a uniform flow leads to acceptable errors on the modal estimation.

In this section, we evaluate the influence of shear flow in our modal detection tool at one specific operating point 

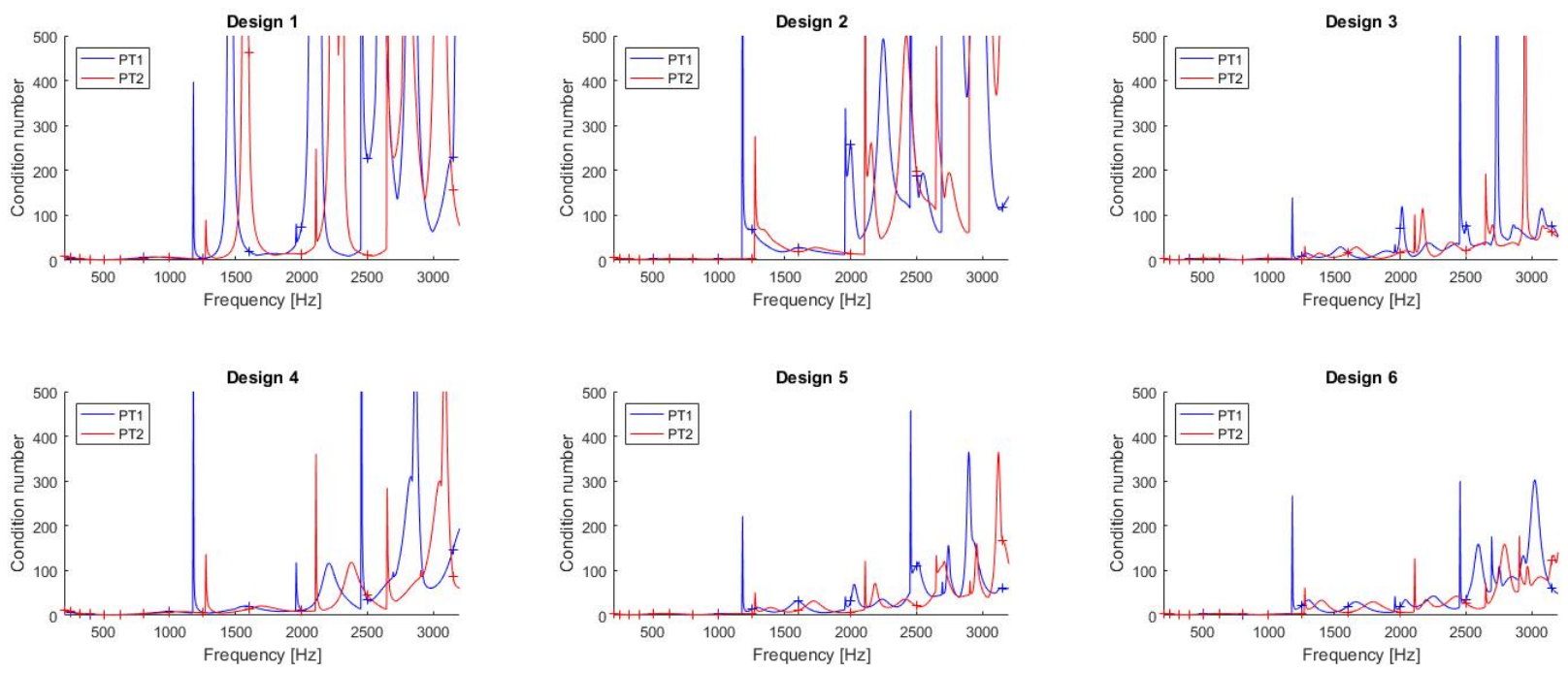

Fig. 4 Condition number obtained under the two operating point conditions, considering each microphone array design defined in this study.

which corresponds to PT2 configuration. The RANS flow has been directly used as a base flow input to compute the modes solving the linearised Euler Equations in MAMOUT, and has been post-treated, to assess the equivalent mean temperature, flow velocity and density to consider under the uniform flow assumption.

As MAMOUT code gives us the radial dependence of modes normalized by its value at the edge $r=R$, equation 2 is slightly modified in:

$$
\hat{p}(x, r, \theta, v)=\sum_{m=-\infty}^{+\infty} \sum_{n=0}^{n=+\infty} \sum_{\varepsilon= \pm} B_{m, n}^{\varepsilon}(v) \frac{f_{m, n}(r)}{f_{m, n}(R)} e^{-\mathrm{i} m \theta} e^{-\mathrm{i} k_{m, n}^{\varepsilon}(v) x}
$$

where $B_{m, n}^{\varepsilon}(v)=A_{m, n}^{\varepsilon}(v) \times f_{m, n}(R)$. The modal basis given under the uniform flow hypothesis (see section II.A) gives the propagation matrix $\mathbf{G}^{\text {uniform }}$. The modal basis (number of cut-on modes and wave number $k_{m, n}^{\varepsilon}$ ) given by MAMOUT under the shear flow hypothesis (see section II.B gives the propagation matrix $\mathbf{G}^{\text {shear }}$. Wave numbers associated to operating condition PT2 are represented in figure 5. The expression given in equation (18) is slightly different as the radial dependence is normalized. Let us denotes $\mathbf{S}^{\text {uniform }}$ and $\mathbf{S}^{\text {shear }}$ as the power cross spectral matrix of the amplitudes $B_{m, n}^{\varepsilon}$ of the modes given by ARMADA under the uniform and the shear flow hypotheses respectively.

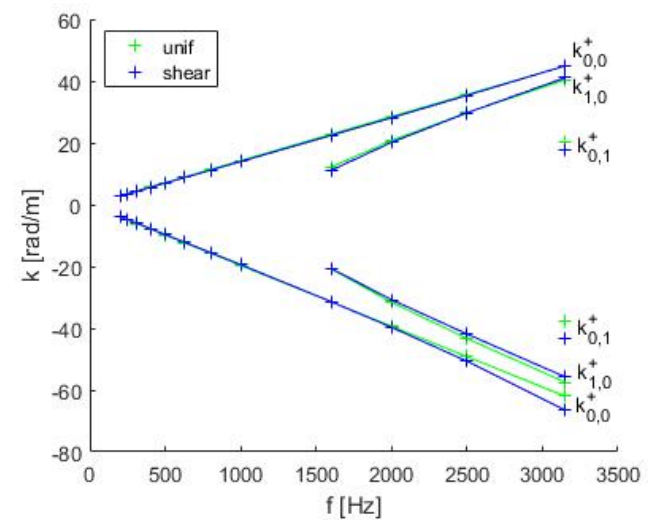

Fig. 5 Wave numbers associated to operating condition PT2 and obtained under the uniform (green) and shear (blue) flow assumptions. 
To evaluate the impact of estimating modes under the uniform flow hypothesis despite the fact that shear flow occurs during measurements, the following procedure is applied:

- A power cross-spectral matrix of the modes amplitude, denoted $\mathbf{S}_{\text {sim }}^{\text {shear }}$, is randomly simulated.

- The modal propagation matrix $\mathbf{G}^{\text {shear }}$ is calculated and the power cross-spectral matrix from wall-flush mounted microphones is evaluated using: $\Gamma^{\text {shear }}=\mathbf{G}^{\text {shear }} \mathbf{S}_{\text {sim }}^{\text {shear }} \mathbf{G}^{\text {shear H}}$. It models the measured power cross-spectral matrix considering a shear flow.

- The quadratic minimization problem 19 is iteratively resolved, considering $\boldsymbol{\Gamma}^{\text {mesure }}=\boldsymbol{\Gamma}^{\text {shear }}$, with the estimated power cross-spectral matrix of the modes amplitude $\tilde{\mathbf{S}}_{\text {est }}^{\text {uniform }}$, as a result.

- The global estimation error (\%) under uniform flow hypothesis is evaluated by:

$$
\epsilon=\frac{\left\|\tilde{\mathbf{S}}_{\text {est }}^{\text {uniform }}-\mathbf{S}_{\text {sim }}^{\text {shear }}\right\|_{\mathrm{F}}}{\left\|\mathbf{S}_{\text {sim }}^{\text {shear }}\right\|_{\mathrm{F}}} \times 100
$$

- For each azimuthal mode $m$ and radial mode $n$, the error percentage between the simulated mode amplitude and the estimated one, under uniform flow hypothesis is evaluated by:

$$
\epsilon_{[m, n, \varepsilon]}=\frac{\left\|\sqrt{\tilde{\mathbf{S}}_{\text {est }[m, n, \varepsilon],[m, n, \varepsilon]}^{\text {uniform }}}-\sqrt{\mathbf{S}_{\text {sim }[m, n, \varepsilon],[m, n, \varepsilon]}^{\text {shear }}}\right\|_{\mathrm{F}}}{\left\|\sqrt{\mathbf{S}_{\text {sim }[m, n, \varepsilon],[m, n, \varepsilon]}^{\text {shear }}}\right\|_{\mathrm{F}}} \times 100
$$

The quantification is made with the microphone array design 6. We first check that the antenna is well suited for modal detection under the shear flow hypothesis, by applying the same numerical evaluation procedure as presented in section [III. The estimation error is always smaller than $1 \%$ regardless the frequency.

The mean, minimum and maximum global estimation errors 222 are represented in Figure 6 , The mean values of the mode amplitude error at each frequency are represented in Figure 7 . For frequency smaller or equal to $1000 \mathrm{~Hz}$, the mode amplitude error varies from $0 \%$ to $15 \%$, with a mean value always smaller than $1 \%$. The mean value of the mode amplitude error is always smaller than $5 \%$ at $1600 \mathrm{~Hz}, 2000 \mathrm{~Hz}$ and $2500 \mathrm{~Hz}$ but become more important at $3150 \mathrm{~Hz}$ $(44 \%)$. At this frequency, the $n=1$ radial mode is cut-on. This mode appears to be very sensitive to refraction effects, which are only taken into account when the modal basis is computed with the shear flow approach.
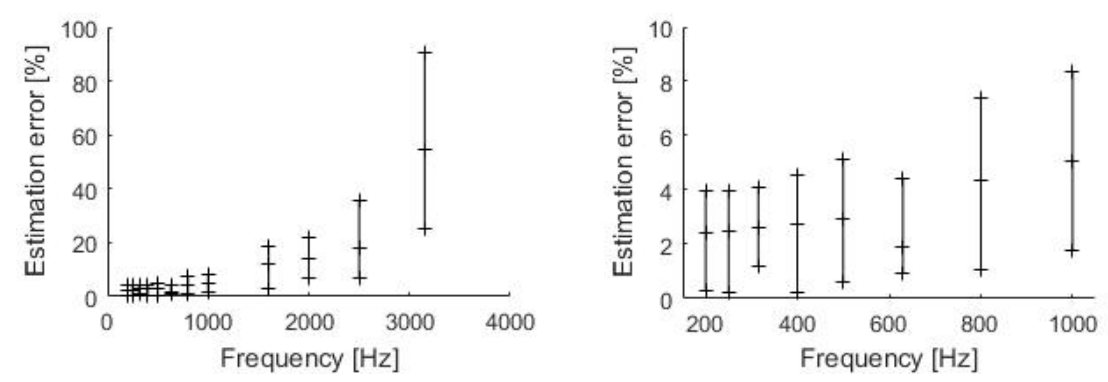

Fig. 6 Mean, minimum and maximum estimation errors (equation (22) obtained with 100 simulations at the central frequency of third octave band between $200 \mathbf{H z}$ and $3150 \mathbf{~ H z ~ ( l e f t ) ~ a n d ~ b e t w e e n ~} 200 \mathbf{H z}$ and $1000 \mathbf{~ H z}$ (right).

As the statistical properties of the power cross spectral matrix of the modes that will occurs during the IDEAS measurements are not known, it is not possible to generalize this concluding remarks, but the potential misleading impact of considering an uniform flow modal basis on the characterization under shear flow conditions should be kept in mind at $3150 \mathrm{~Hz}$.

\section{Experimental setup}

The test bench simulates a new concept of air conditioning system constituted by a jet pump. There is composed of an inlet, a mixing part where the jet is and a diffuser part (Figure 8 left). After the diffuser outlet, the duct is cylindrical 


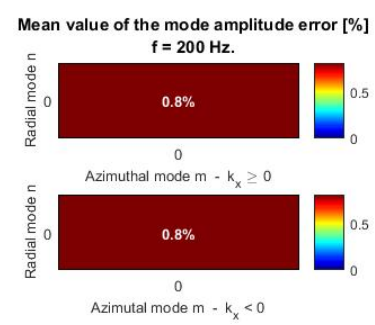

Mean value of the mode amplitude error $[\%]$

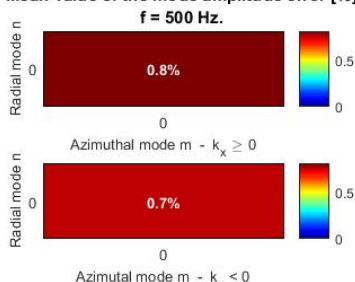

Azimutal mode $m-k_{x}<0$

Mean value of the mode amplitude error [\%]

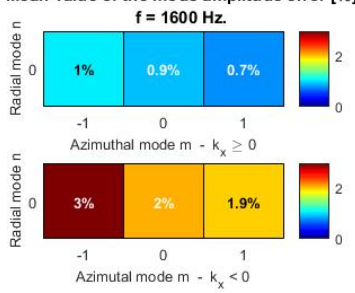

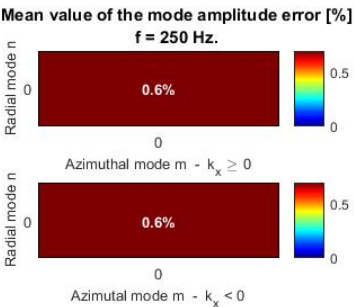

Mean value of the mode amplitude error [\%]

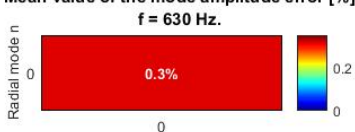

Azimuthal mode $m-k_{x} \geq 0$

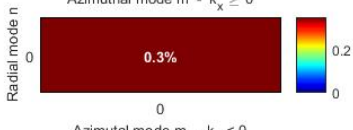

Azimutal mode $m-k_{x}<0$

Mean value of the mode amplitude error $[\%]$

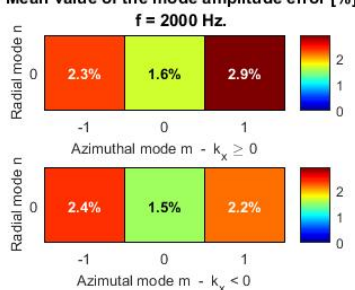

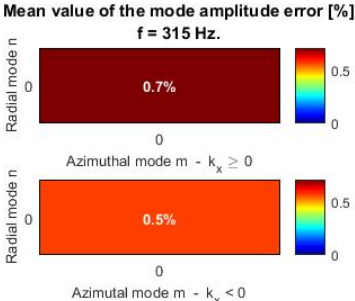

Mean value of the mode amplitude error [\%]

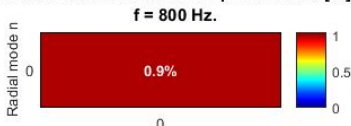

Azimuthal mode $m-k_{x} \geq 0$

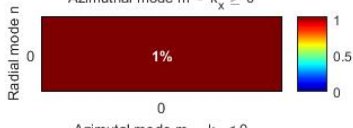

Azimutal mode $m-k_{x}<0$

Mean value of the mode amplitude error [\%]

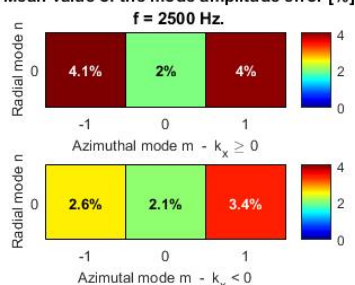

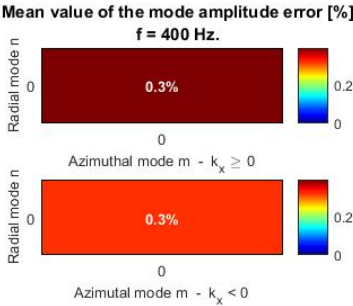

Mean value of the mode amplitude error [\%]

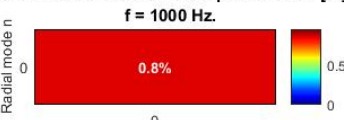

Azimuthal mode $m-k \geq 0$

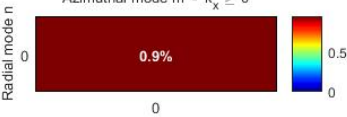

Mean value of the mode amplitude error [\%]

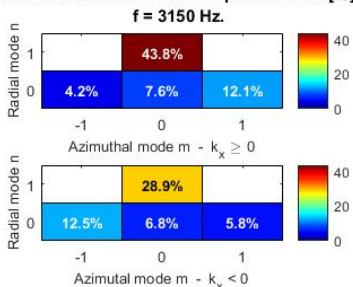

Fig. 7 Mean value of the mode amplitude error (equation (23)) obtained with 100 simulations at the central frequency of third octave band between $200 \mathrm{~Hz}$ and $3150 \mathrm{~Hz}$.

(diameter of $173 \mathrm{~mm}$ - Figure 8 center) up to the anechoic chamber where the termination is flush to the wall (Figure 8 right). The ONERA modal detection ring take place $2.41 \mathrm{~m}$ from the jet exhaust, after about $1.66 \mathrm{~m}$ of cylindrical part. The ring has been instrumented with $29^{1 / 4}$ " condenser microphones Brüel\&Kjaer. As they are flush mounted with grid, pressure-field microphones type 4938, were chosen. Two sections of the ring (upstream and downstream) have been also instrumented with each three pressure taps in order to check the aerodynamic variation along the ring axial extent.
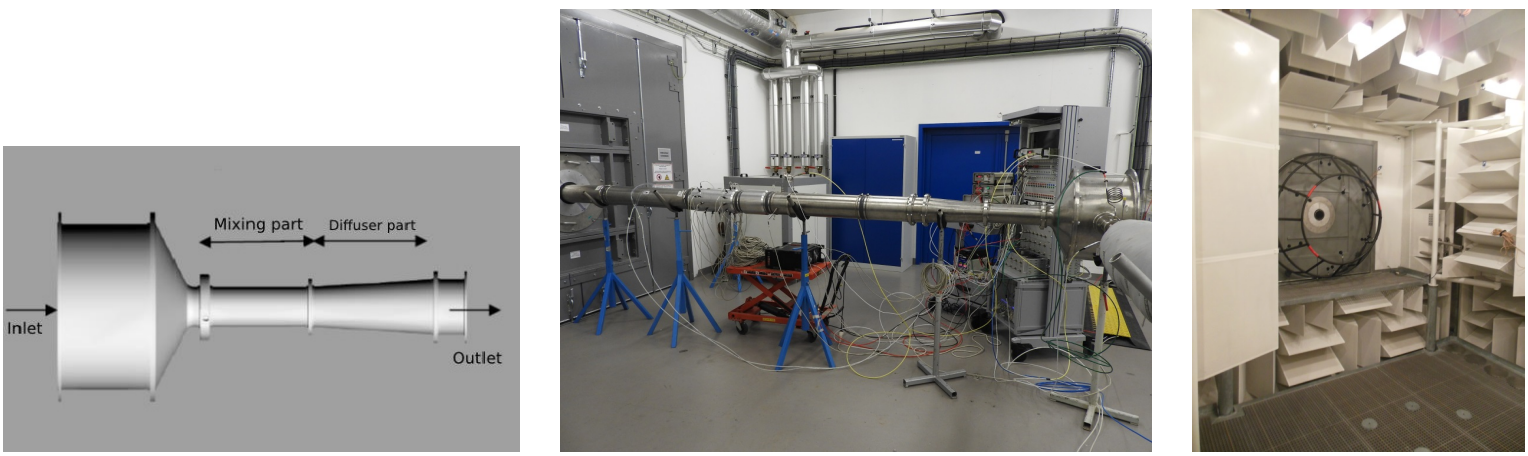

Fig. 8 Jet-pump geometry (left) - Photography of Liebherr's test bench (center) and termination in the anechoic chamber (right).

The signals are acquired with a B\&K data acquisition system LANXI that allows synchronization accuracy between channel less than $20 \mathrm{~ns}$. The sampling frequency was fixed at $32768 \mathrm{~Hz}$ and a high-pass filter was set at $22.4 \mathrm{~Hz}$. Thirty seconds of signals were recorded, that allows to perform spectral analysis every $8 \mathrm{~Hz}$ with 240 averages. Different jet operating conditions of the pump are studied during the test, with $c_{0}$ varying from 335 to $355 \mathrm{~m} \cdot \mathrm{s}^{-1}$ and $M_{0}$ from 0.05 
to 0.34 . We focus on the ones that are closest to the specification conditions:

- PTMes1: Speed of sound $352 \mathrm{~m} \cdot \mathrm{s}^{-1}$ and Mach 0.25

- PTMes2: Speed of sound $346 \mathrm{~m} \cdot \mathrm{s}^{-1}$ and Mach 0.19

Those values are provided by Liebherr Aerospace, based on temperature, pressure and mass flow rate measurements in the area of the microphones array. Unfortunately, the conditions that were considered in the preliminary study were not reached. Nevertheless, the suitability of the antenna design for modal deconvolution is preserved (see Figure 9). Let us notice that the condition number diverges in the vicinity of the modes cut-on frequency. Reproducibility test points are done.

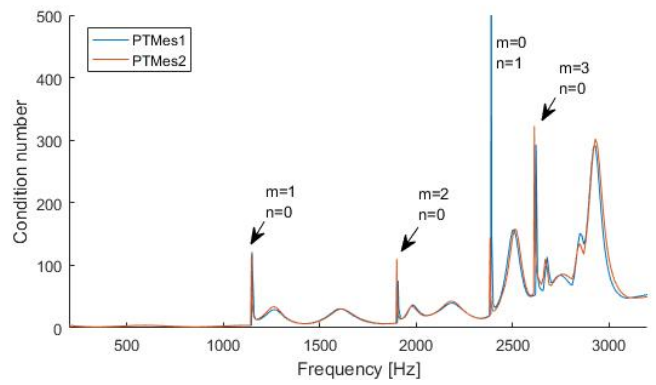

Fig. 9 Condition number obtained under the operating conditions met during the test.

Azimuthal decomposition using the twelve microphones of the upstream ring of the array was process in real time as well as a first analysis of the complete radial and azimuthal decomposition using the deconvolution method. This work was done for the twelve central frequency of the third octave band between $200 \mathrm{~Hz}$ and $3150 \mathrm{~Hz}$ which is the band of interest for the liner characterizations. A post-processing of the data is also done for the frequencies of the entire bandwith between $200 \mathrm{~Hz}$ and $3150 \mathrm{~Hz}$, with a frequency step of $8 \mathrm{~Hz}$. Azimuthal Fourier transform allows us to determine the azimuthal mode amplitudes as a function of $x$ and $v$, for azimuthal order $m$ varying from -5 to 5 :

$$
\left|\frac{1}{2 \pi} \int_{0}^{2 \pi} \hat{p}(x, R, \theta, v) \times e^{\mathrm{i} m_{0} \theta} d \theta\right|=\left|A_{m_{0}}(x, R, v)\right|
$$

The azimuthal mode amplitudes can also be recalculated from the power cross-spectral matrix of the amplitudes of the mode obtained by modal deconvolution:

$$
\left|A_{m_{0}}(x, R, v)\right|^{2} \approx \sum_{n_{1}, n_{2}=0}^{+\infty} \sum_{\varepsilon_{1}, \varepsilon_{2}= \pm} \mathbf{S}_{\left[m_{0}, n_{1}, \varepsilon_{1}\right],\left[m_{0}, n_{2}, \varepsilon_{2}\right]}(v) J_{m_{0}}\left(\mu_{m_{0}, n_{1}}\right) J_{m_{0}}\left(\mu_{m_{0}, n_{2}}\right) e^{-\mathrm{i}\left[k_{m_{0}, n_{1}}^{\varepsilon}(v)-k_{m_{0}, n_{2}}^{\varepsilon}(v)\right] x}
$$

Azimuthal mode amplitudes obtained by those two methods are very closed (see Figure 10, except in the vicinity of the cut-on modes frequencies. The part of the measured cross-spectral matrix that is not explained by the estimated PSCM of the amplitudes of the mode is rather small, as represented in Figure 11. This leads us to have confidence in the results obtained by ARMADA on the basis of the measurements made with the microphones array designed in the preliminary study. 

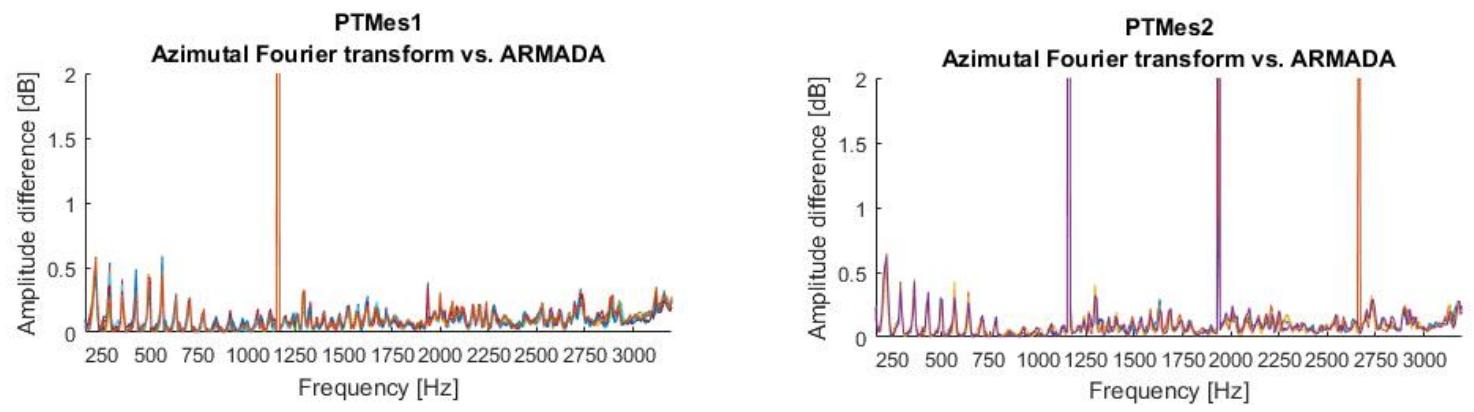

Fig. 10 Discrepancy between the azimuthal mode amplitude obtained by equations (24) and (25) under the operating conditions PTMes1 (left) and PTMes2 (right).
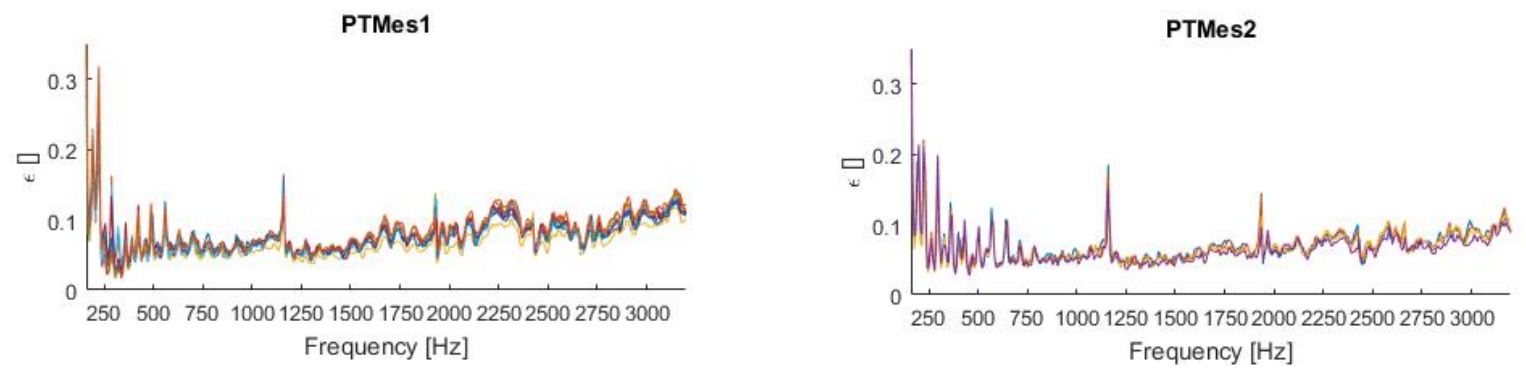

Fig. 11 Part of the data that is not explained by the power cross spectral matrix of the amplitudes of the mode estimated by ARMADA, under the operating conditions PTMes1 (left) and PTMes2 (right).

In a rigid cylindrical duct, the sound intensity carried by the azimuthal mode $m$ and radial mode $n$ propagating in the direction given by $\varepsilon$ may be expressed by [9]:

$$
I_{m, n}^{\varepsilon}(v)=\frac{1}{\rho_{0} c_{0}}\left|A_{m, n}^{\varepsilon}(v)\right|^{2} Y_{m, n} \frac{k_{0}(v)\left[\beta_{0}^{2} k_{m, n}^{\varepsilon}(v)+M_{0} k_{0}(v)\right]}{\left[k_{0}(v)-M_{0} k_{m, n}^{\varepsilon}(v)\right]^{2}}
$$

where $\rho_{0}$ is the air density and $Y_{m, n}$ is given by:

$$
Y_{m, n}= \begin{cases}\frac{1}{2} & \text { if } m=n=0 ; \\ \frac{1}{2}\left(1-\frac{m^{2}}{\mu_{m, n}^{2}}\right) J_{m, n}^{2}\left(\mu_{m, n}\right) & \text { otherwise }\end{cases}
$$

The upstream and downstream sound intensity are given by adding up the modal intensity of all modes propagating upstream and downstream respectively. Let's take a look at the mean, maximum and minimum values of the upstream modal intensity obtained on the different measurement points related to PTMes1 and PTMes2. Results, normalized by the upstream sound intensity, are given in Figures 12 and 13 The two jet conditions PtMes1 and PtMes2 present very similar results. The $m=0$ and $n=0$ mode is predominant. The $m=0$ and $n=1$ mode, which appeared to be the most sensitive to refraction effects (see section IV], is not very energetic. The values are relatively constant from one measurement to another, except for the azimuthal mode 3 . One can also notice that the positive and negative value of the same azimuthal order $(m= \pm 1, \pm 2$ and \pm 3 ) have equivalent intensity, which could be understandable as for jet noise application no preferential rotating sense propagation is expected. The positive and negative azimuthal mode are correlated. 


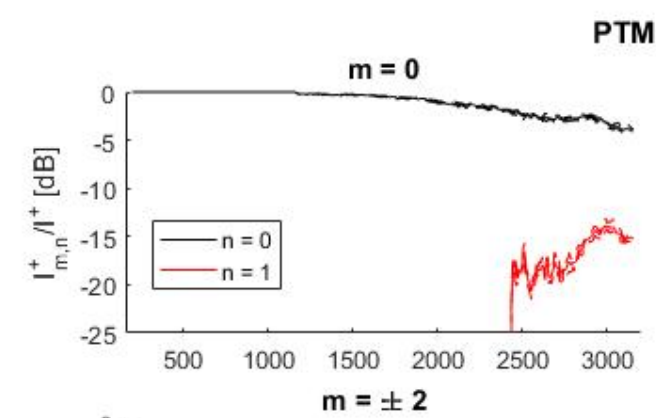

TMes1
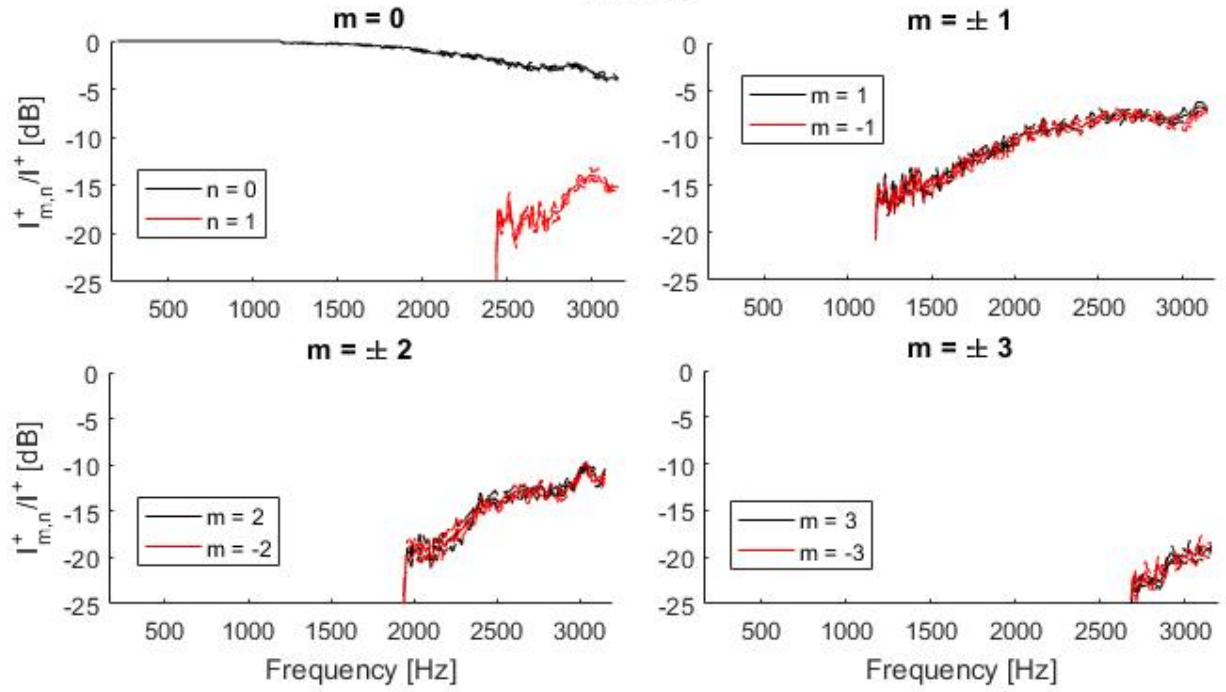

Fig. 12 Normalized mean, maximum and minimum values of the upstream modal intensity obtained under the operating condition PTMes1.

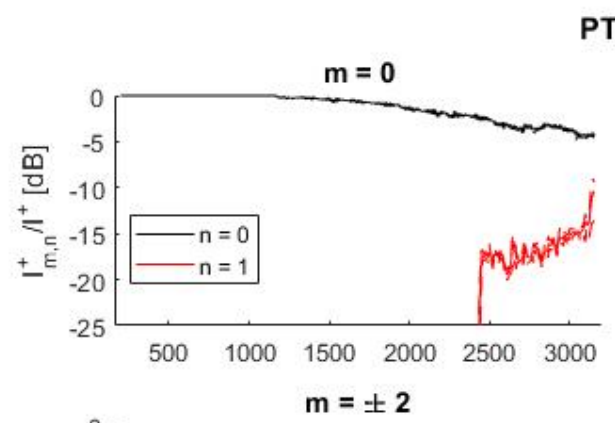

PTMes2
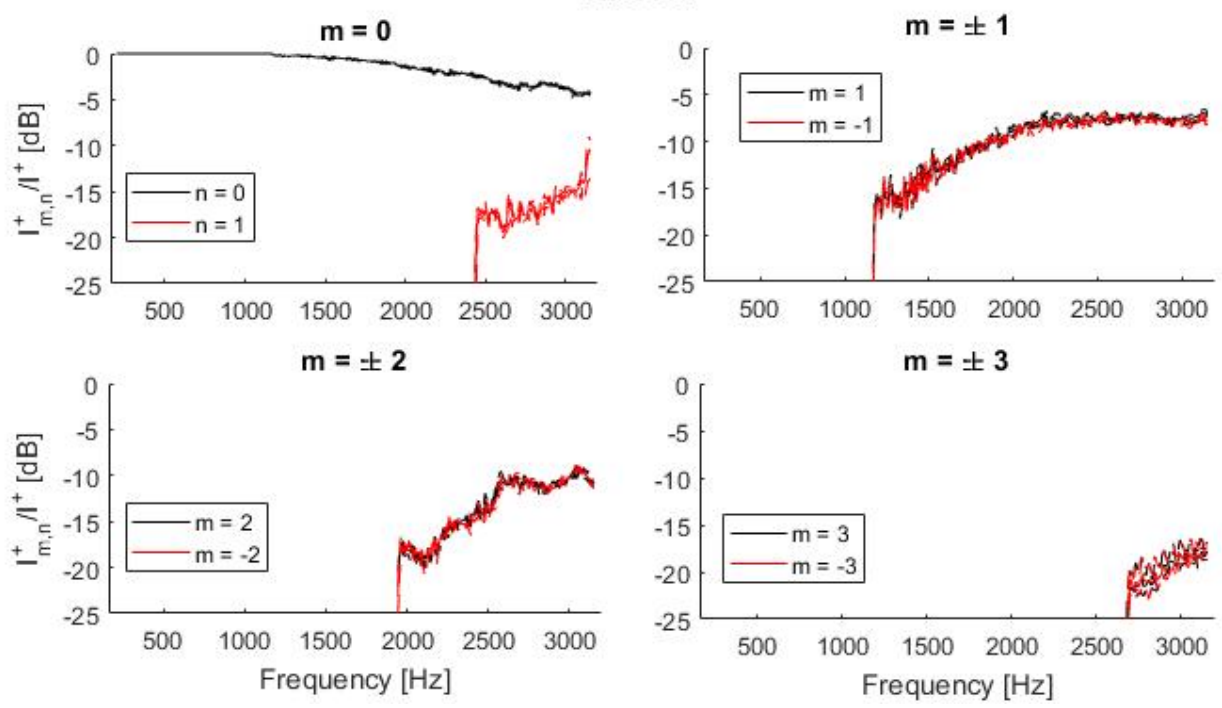

Fig. 13 Normalized mean, maximum and minimum values of the upstream modal intensity obtained under the operating condition PTMes2.

The reflexion coefficient associated to azimuthal modes are represented in Figures 14 and 15 . It is defined as the ratio between the downstream and the upstream azimuthal intensity, where $I_{m_{0}}^{\varepsilon}$ is calculated by summation of all $I_{m_{0}, n}^{\varepsilon}$. In the vicinity of the cut-on frequency, upstream and downstream mode intensities are equivalent, which exhibit important reflection. When the frequency increases and therefore deviates from the cut-on frequency, the upstream contribution strongly decreases. Once again, the two jet conditions PtMes1 and PtMes2 present very similar results, with a reflection coefficient slightly higher in the PTMes2 condition. Such a reflection implies non-zero correlation coefficients between upstream and downstream modes, that also justify the correlated mode assumption. 

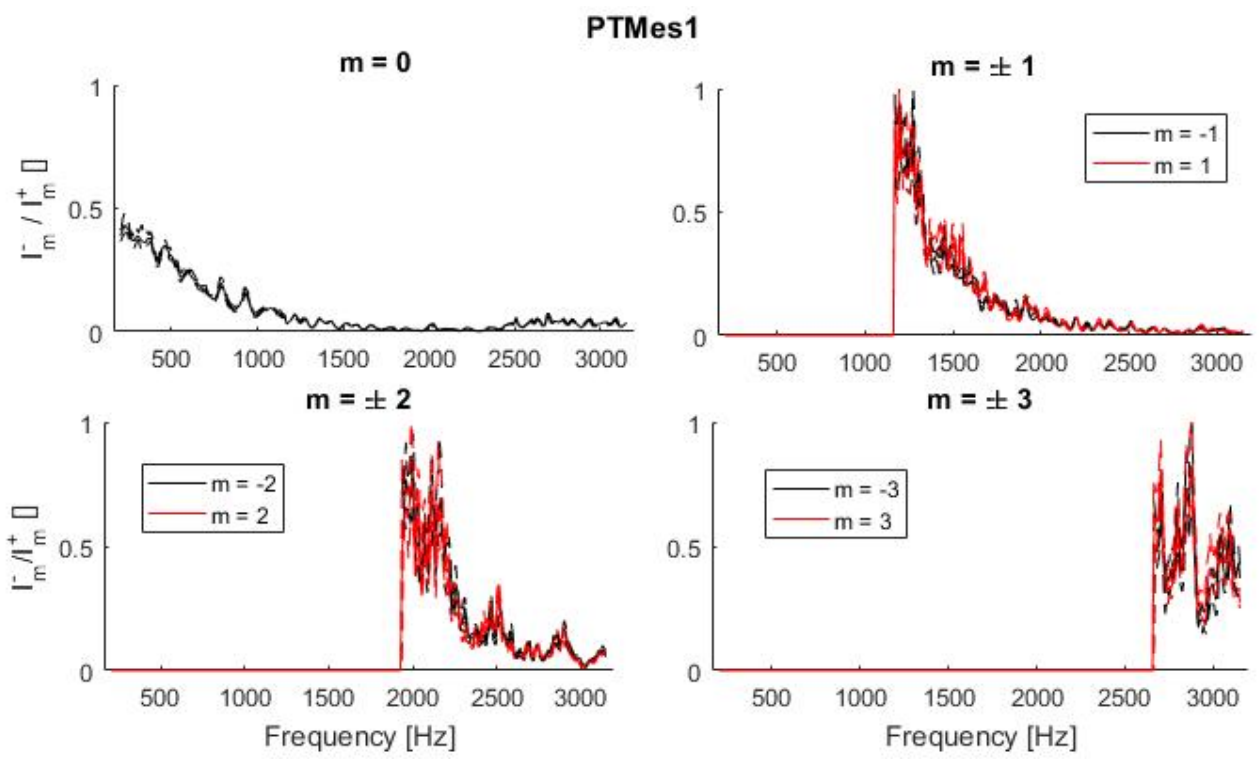

Fig. 14 Normalized mean, maximum and minimum values of the azimuthal mode reflection obtained under the operating condition PTMes1.
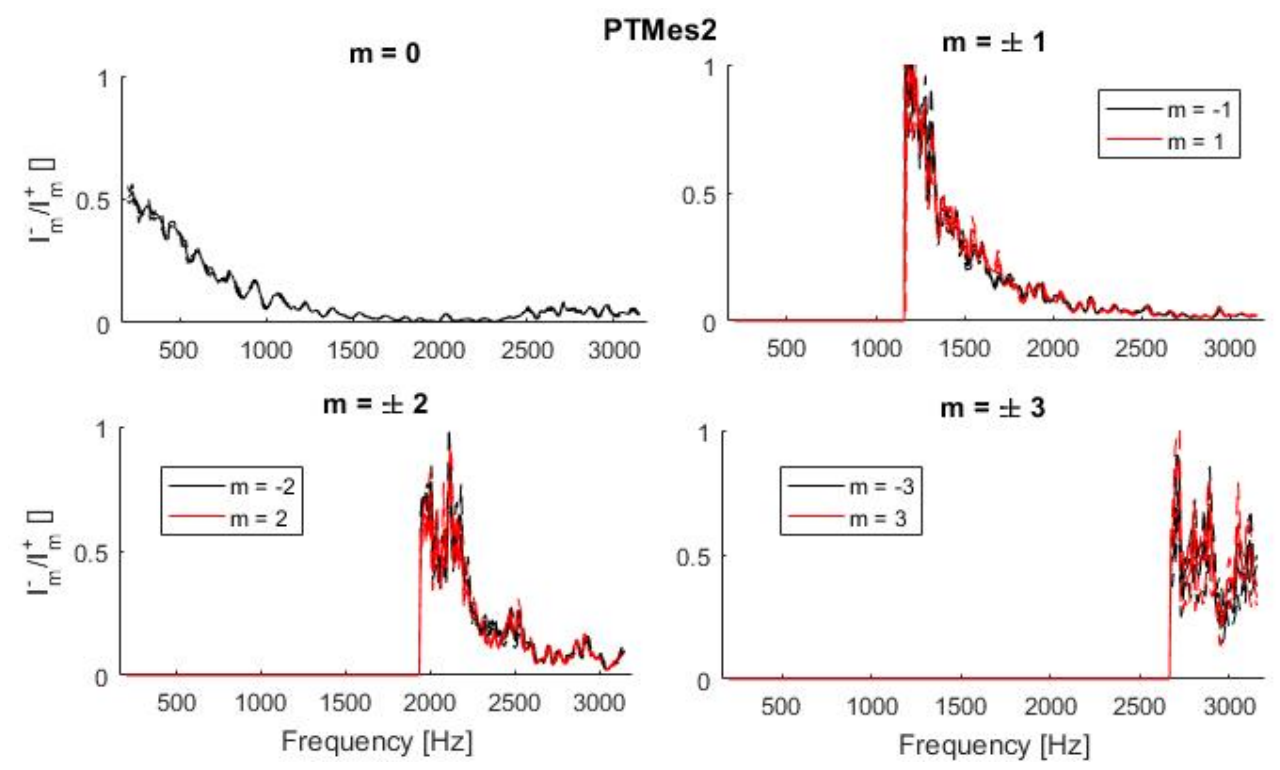

Fig. 15 Normalized mean, maximum and minimum values of the azimuthal mode reflection obtained under the operating condition PTMes2.

Finally, this first experimental campaign validates the capability of the completed methodology for the characterization of the modal content of the jet pump noise. Measurements with acoustic liners will be performed in the near future. At that time, it would be interesting to also make measurements without acoustic liners in such a way that we can apply our deconvolution method under the shear flow hypothesis, in order to validate (or not...) the theoretical sensitivity analysis presented in section IV. 


\section{Conclusion}

In the first part of this article, a modal detection tool in presence of uniform as well as shear flow has been introduced. Based on the ONERA's deconvolution method ARMADA developed for infinite circular rigid duct of constant section in presence of uniform flow, it uses modal shapes given by MAMOUT code in case of shear flow. Only cut-on modes are considered. The modal detection network will be implemented in a hard-wall region of the duct with circular and constant section, located downstream of the diffuser, far from the lined walls. Hypotheses of circular rigid duct of constant radius are, then, verified. Correlations are taken into account in the estimation tool, as wave reflections may occur at the end of Liebherr's bench.

In the second part, the design of the IDEAS microphone array has been defined, considering uniform flow only. In the frequency bandwidth of interest and under the operating conditions, the azimuthal order of cut-on modes varies from -3 to 3, with a maximum of 2 radial modes. Different sensor arrangements have been defined and compared in terms of condition number associated to the linear problem our deconvolution method have to solve. Composed by 29 microphones, the retained design allows an accurate theoretical estimation of the correlated modal content of the acoustic pressure field for the two operating points, at the central frequency of third octave bands between 200 and 3150 $\mathrm{Hz}$.

In the third part, the influence of shear flow in our modal detection tool under the second operating point condition is evaluated. The microphone array defined in section III is well suited for modal detection under the shear flow hypothesis. The impact of estimating modes under the uniform flow hypothesis despite the fact that shear flow occurs during measurements has been evaluated. As the statistical properties of the power cross spectral matrix of the modes that will occur during the IDEAS measurements are not known, it is not possible to generalize this study, but considering an uniform flow modal basis to characterize the modal content of the acoustic pressure field produced under shear flow conditions has a minor impact in most cases for frequencies smaller than $2500 \mathrm{~Hz}$ but can become important at $3150 \mathrm{~Hz}$.

Finally, the experimental setup has been introduced. Analysis of the measurements performed in the facility without use of acoustic liners has been detailed for two operating conditions. The part of the measured cross-spectral matrix that is not explained by the ARMADA estimated modes is rather small. Furthermore, azimuthal mode amplitudes are very closed from the one obtained by azimuthal Fourier transform. This leads us to have confidence in the results obtained by ARMADA on the basis of the measurements made with the microphones array designed in the preliminary study. The two jet conditions present very similar results. The $m=0$ and $n=0$ mode is predominant. Positive and negative values of the same azimuthal order have equivalent intensity, which could be understandable as for jet noise application no preferential rotating sense propagation is expected. Important reflection coefficients have been exhibit in the vicinity of the associated cut-on frequency, that strongly decreases as the frequency increases. Correlation occurs between upstream and downstream modes as well as positive and negative azimuthal modes, that justify the correlated mode assumption. Measurements with acoustic liners will be performed in the near future.

\section{Acknowledgement}

This project has received funding from the Clean Sky 2 Joint Undertaking under the European Union's Horizon 2020 research and innovation programme under grant agreement No 716499. This study was conducted in collaboration with Liebherr Aerospace, and with the participation of ATECA for the manufacturing of the ONERA modal detection ring. Finally, the authors would like to thank Laurent Coste from ONERA and Mathieu Albert from Liebherr Aerospace for their involvement during the experimental campaign.

\section{References}

[1] Fauqueux, S., and Davy, R., "Modal deconvolution method in a finite circular duct, using flush-mounted microphones," 24th AIAA/CEAS Aeroacoustics Conference, AIAA, Atlanta, Georgia, 2018. doi:10.2514/6.2018-3927.

[2] Rienstra, S., and Hirschberg, A., An Introduction to Acoustics, Eindhoven University of Technology, 2017. https://www . win. tue.nl/ sjoerdr/papers/boek.pdf

[3] Ingard, U., "Influence of fluid motion past a plane boundary on sound reflection, absorption, and transmissions," J. Acoust. Soc. Am., Vol. 31, No. 1035, 1959. doi:10.1121/1.1907805.

[4] Myers, M., "On the acoustic boundary condition in the presence of flow," Journal of Sound and Vibration, Vol. 71, 1980, pp. 429-434. 
[5] Boyer, G., Brazier, J.-P., and Piot, E., "Theoretical investigation of hydrodynamic surface mode in a lined duct with sheared flow and comparison with experiment," Journal of Sound and Vibrathion, Vol. 330, No. 8, 2011, pp. 1793-1809.

[6] Welch, P., "The use of Fast Fourier Transform for the estimation of power spectra : A method based on time averaging over short, modified periodograms," IEEE Transactions on Audio and Electroacoustics, Vol. 15, No. 2, 1967, pp. 70-73. doi:10.1109/TAU.1967.1161901.

[7] Fleury, V., and Davy, R., "Analysis of jet-airfoil interaction noise sources by using a microphone array technique,” 2016 , pp. 44-66. doi:https://doi.org/10.1016/j.jsv.2015.11.027.

[8] Tapken, U., and Enghardt, L., "Optimization of sensor arrays for radial mode analysis in flow ducts," 12th AIAA/CEAS Aeroacoustics Conference, AIAA, Cambridge, Massachusetts, 2006. doi:10.2514/6.2006-2638.

[9] Morfey, C., “Acoustic energy in non-uniform flows,” Journal of Sound and Vibrathion, Vol. 14, No. 2, 1971 , pp. 159-170. 